\title{
UCRL-BOOK-201405
}

LAW RENCE LIVERMORE N A T IO N A L LABORATORY

\section{Modeling the Reactions of Energetic Materials in the Condensed Phase}

L. E. Fried, M. R. Manaa, J. P. Lewis

December 11, 2003

Overviews of Recent Reseaarch on Energetic Materials 
This document was prepared as an account of work sponsored by an agency of the United States Government. Neither the United States Government nor the University of California nor any of their employees, makes any warranty, express or implied, or assumes any legal liability or responsibility for the accuracy, completeness, or usefulness of any information, apparatus, product, or process disclosed, or represents that its use would not infringe privately owned rights. Reference herein to any specific commercial product, process, or service by trade name, trademark, manufacturer, or otherwise, does not necessarily constitute or imply its endorsement, recommendation, or favoring by the United States Government or the University of California. The views and opinions of authors expressed herein do not necessarily state or reflect those of the United States Government or the University of California, and shall not be used for advertising or product endorsement purposes. 


\title{
Modeling the Reactions of Energetic Materials in the
}

\section{Condensed Phase}

\author{
Laurence E. Fried, M. Riad Manaa \\ Lawrence Livermore National Laboratory
}

And

James P. Lewis

Department of Physics and Astronomy

Brigham Young University

N319 ESC, P.O. Box 24658

Provo, UT 84602-4658 


\section{INTRODUCTION}

High explosive (HE) materials are unique for having a strong exothermic reactivity, which has made them desirable for both military and commercial applications. Although the history of HE materials is long, condensed-phase properties are poorly understood. Understanding the condensed-phase properties of HE materials is important for determining stability and performance. Information regarding HE material properties (for example, the physical, chemical, and mechanical behaviors of the constituents in plasticbonded explosive, or PBX, formulations) is necessary in efficiently building the next generation of explosives as the quest for more powerful energetic materials (in terms of energy per volume) moves forward. In addition, understanding the reaction mechanisms has important ramifications in disposing of such materials safely and cheaply, as there exist vast stockpiles of HE materials with corresponding contamination of earth and groundwater at these sites, as well as a military testing sites (for example, see Refs. ${ }^{1}$ and $\left.{ }^{2}\right)$.

In modeling HE materials there is a need to better understand the physical, chemical, and mechanical behaviors from fundamental theoretical principles. Among the quantities of interest, for example, in plastic-bonded explosives (PBXs), are thermodynamic stabilities, reaction kinetics, equilibrium transport coefficients, mechanical moduli, and interfacial properties between HE materials and the polymeric binders. These properties are needed, generally as a function of stress state and temperature, for the development of improved micro-mechanical models, which represent the composite at the level of grains and binder. Improved micro-mechanical models are needed to describe the response of PBXs to dynamic stress and/or thermal loading to 
yield information from which constitutive laws for use in continuum modeling approaches can be formulated and/or parameterized.

Detailed description of the chemical reaction mechanisms of condensed energetic materials at high densities and temperatures is essential for understanding events that occur at the reactive front of these materials under combustion or detonation conditions. Under shock conditions, for example, energetic materials undergo rapid heating to a few thousand degrees and are subjected to a compression of hundreds of kilobars, ${ }^{3}$ resulting in almost 30\% volume reduction. Complex chemical reactions are thus initiated, in turn releasing large amounts of energy to sustain the detonation process. Clearly, understanding of the various chemical events at these extreme conditions is essential in order to build predictive models of material properties that can be incorporated into fullycontinuum approaches of describing the detonation process at the macroscopic level.

Scientific investigation into the reactive process has been undertaken over the past two decades. However, the sub- $\mu$ s time scale associated with explosive reactions in addition to the highly exothermic conditions associated with an explosion make experimental investigation of the decomposition pathways difficult at best. Elucidating decomposition pathways is further complication due to the increased instability of HE materials under high temperature and pressure regimes.

More recently, new computational approaches to energetic material reactivity have been developed. Furthermore, methods to model condensed-phase reaction pathways have only recently been developed. The doubling of computational power approximately every 18 months has made atomistic condensed-phase modeling more feasible. Here we focus on two different aspects of condensed-phase reaction modeling in 
terms of chemical equilibrium methods and atomistic modeling of condensed-phase reactions. These are complimentary approaches to understanding the chemical reactions of high explosives. Chemical equilibrium modeling uses a highly simplified thermodynamic picture of the reaction process. This leads to a convenient and predictive model of the detonation process. Chemical equilibrium codes are often used in the design of new materials, both at the level of synthesis chemistry and formulation.

Atomistic modeling, on the other hand, is a rapidly emerging area. Atomistic calculations employ far fewer empirical parameters than chemical equilibrium calculations. Nevertheless, the atomistic modeling of chemical reactions requires an accurate global Born-Oppenheimer potential energy surface. Traditionally, such a surface is constructed numerically by solving the Schrödinger equation at each configuration in a molecular dynamics simulation. Recent developments, however, indicate that the use of reactive classical potentials may also be a viable option in studying condensed phase reactions.

Chemical reactions may also occur through diabatic processes involving several electronic states. The importance of such reactions on the overall chemistry of energetic materials is still a matter of debate. We review recent work on the effect of high pressure and defects in modifying the excited electronic states of energetic materials.

\section{CHEMICAL EQUILIBRIUM}

The energy content of an energetic material often determines its practical utility. An accurate estimate of the energy content is essential in the design of new materials ${ }^{4}$ and in the understanding of quantitative detonation tests ${ }^{5}$. The useful energy content is 
determined by the anticipated release mechanism. Since detonation events occur on a $\mu \mathrm{s}$ timeframe, any chemical reactions slower than this are not relevant when considering a detonation. Thermodynamic cycles are a useful way to understand energy release mechanisms. Detonation can be thought of as a cycle that transforms the unreacted explosive into stable product molecules at the Chapman-Jouget state ${ }^{6}$ (see Figure 1). This is simply described as the slowest steady shock state that conserves mass, momentum, and energy. Similarly, the deflagration of a propellant converts the unreacted material into product molecules at constant enthalpy and pressure. The nature of the Chapman-Jouget and other special thermodynamic states important to energetic materials is strongly influenced by the equation of state of stable detonation products.

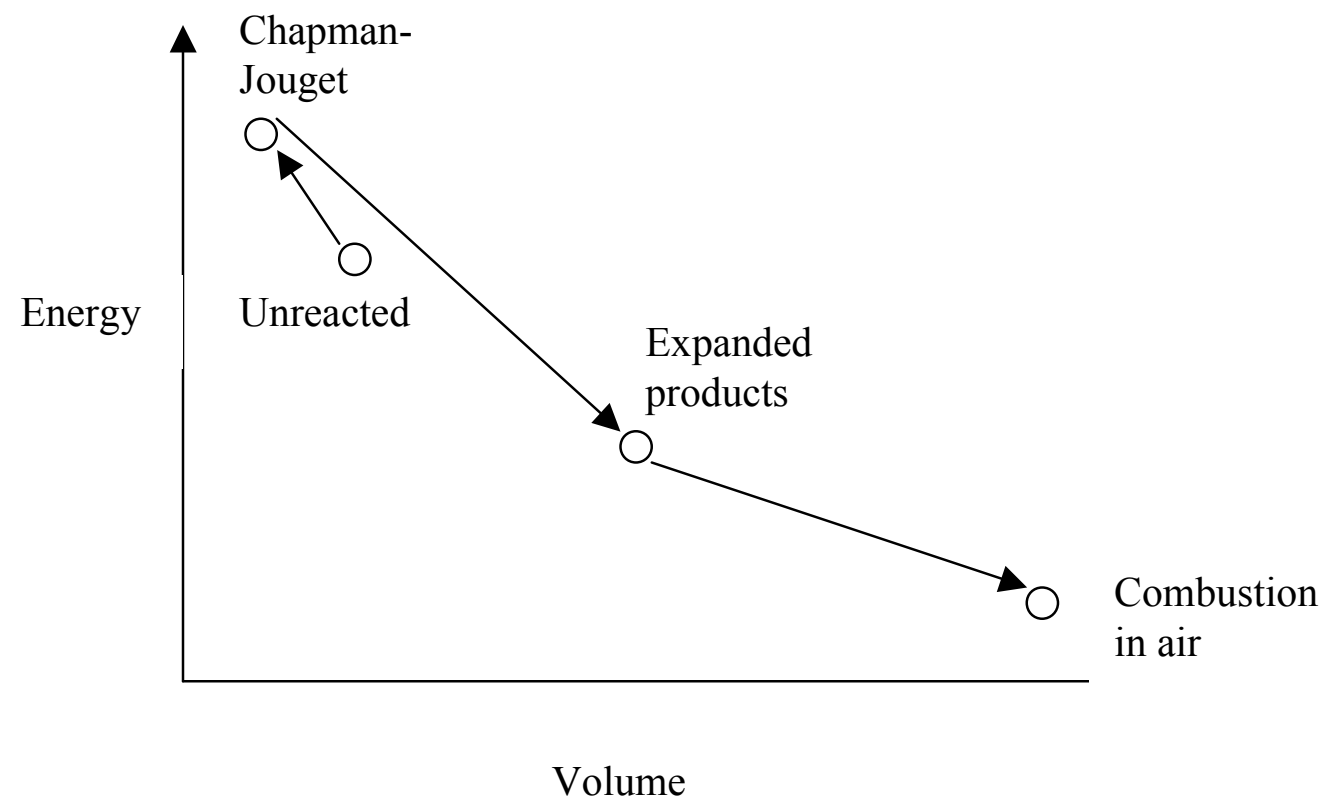

Figure 1: A thermodynamic picture of detonation.

Understanding energy release in terms of thermodynamic cycles ignores the important question of the time scale of reaction. The kinetics of even simple molecules 
under high-pressure conditions is not well understood. Diamond anvil cell and shock experiments promise to provide insight into chemical reactivity under extreme conditions.

Despite the importance of chemical kinetic rates, chemical equilibrium is often nearly achieved when energetic materials react. This is a consequence of the high temperatures produced by such reactions (up to $6000 \mathrm{~K}$ ). We will begin our discussion by examining thermodynamic cycle theory as applied to high explosive detonation. This is a current research topic because high explosives produce detonation products at extreme pressures and temperatures: up to $40 \mathrm{GPa}$ and $6000 \mathrm{~K}$. Relatively little is known about material equations of state under these conditions. Nonetheless, shock experimentation on a wide range of materials has generated sufficient information to allow reasonably reliable thermodynamic modeling to proceed.

One of the attractive features of thermodynamic modeling is that it requires very little information regarding the unreacted energetic material. The elemental composition, density, and heat of formation of the material are the only information needed. Since elemental composition is known once the material is specified, only density and heat of formation needs to be predicted.

Chapman-Jouget (C-J) detonation theory ${ }^{6}$ implies that the performance of an explosive is determined by thermodynamic states--the Chapman-Jouget state and the connected expansion adiabat, illustrated in Figure 1. Upon expansion, more energy can be released from the combustion of the detonation products, which are often rich in carbon, with the surrounding air. 
Thermochemical codes use thermodynamics to calculate these states, and hence obtain a prediction of explosive performance. The allowed thermodynamic states behind a shock are intersections of the Rayleigh line (expressing conservation of mass and momentum), and the shock Hugoniot (expressing conservation of energy). The C-J theory states that a stable detonation occurs when the Rayleigh line is tangent to the shock Hugoniot.

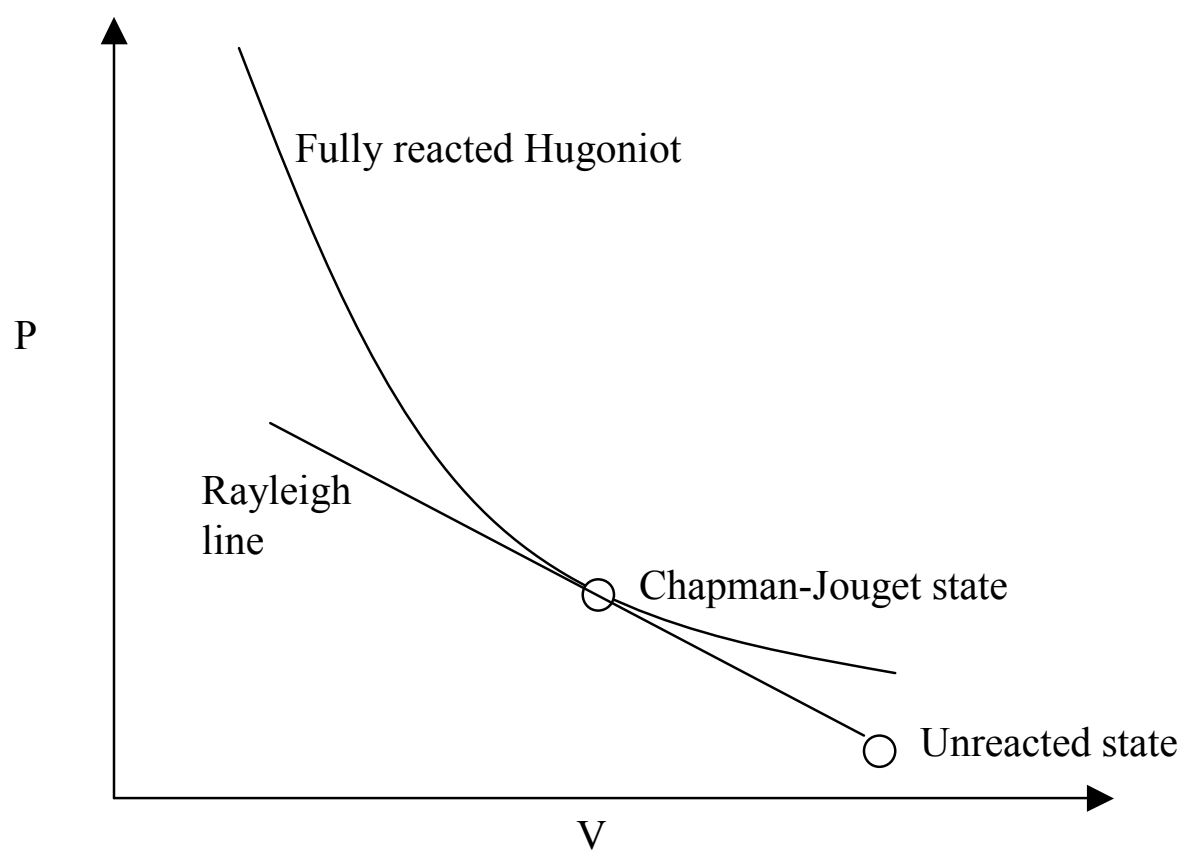

Figure 2: Allowed thermodynamic states in detonation are constrained to the shock Hugoniot. Steady shock waves follow the Rayleigh line.

This point of tangency can be determined, assuming that the equation of state $\mathrm{P}=$ $\mathrm{P}(\mathrm{V}, \mathrm{E})$ of the products is known. The chemical composition of the products changes with the thermodynamic state, so thermochemical codes must simultaneously solve for state variables and chemical concentrations. This problem is relatively straightforward, given that the equation of state of the fluid and solid products is known. 
One of the most difficult parts of this problem is accurately describing the equation of state of the fluid components. Despite its simplicity and lack of rigorous derivation, the Becker-Kistiakowski-Wilson (BKW) ${ }^{7}$ equation of state (EOS) is used in many practical energetic material applications. There have been a number of different parameter sets proposed for the BKW EOS ${ }^{8-11}$. Kury and Souers ${ }^{5}$ have critically reviewed these equations of state by comparing their predictions to a database of detonation tests. They concluded that BKW equations of state could not adequately model the detonation of a copper lined cylindrical charge. The BKWC parameter set ${ }^{12}$ partially overcame this deficiency through more aggressive parameterization techniques. BKWC is not reliable when applied to explosives very high in hydrogen content, however.

It has long been recognized that the highly empirical nature of the BKW equation of state limits the accuracy achievable in detonation calculations. This is particularly important when designing new materials that may have unusual elemental compositions. Efforts to achieve better equations of state have largely been based on the concept of model potentials. With model potentials, molecules interact via idealized spherical pair potentials. Statistical mechanics is then employed to calculate the equation of state of the interacting mixture of effective spherical particles. Most often, the exponential-6 potential is used for the pair interactions:

$\boldsymbol{V}(\boldsymbol{r})=\frac{\varepsilon}{\alpha-6}\left[6 \exp \left(\alpha-\alpha \boldsymbol{r} / \boldsymbol{r}_{m}\right)-\alpha\left(\boldsymbol{r}_{m} / \boldsymbol{r}\right)^{6}\right]$

Here, $r$ is the distance between particles. $r_{\mathrm{m}}$ is the minimum of the potential well. $\varepsilon$ is the well depth, and $\alpha$ is the softness of the potential well. 
The JCZ3 EOS was the first successful model based on a pair potential that was applied to detonation ${ }^{13}$. This EOS was based on fitting Monte Carlo simulation data to an analytic functional form. Ross proposed a soft-sphere perturbation theory for the equation of state of the exponential-6 fluid ${ }^{14}$. Ross, Ree, and others successfully applied this equation of state to detonation and shock problems ${ }^{15-18}$. Kang et al. also derived a fluid perturbation theory designed to work at high-density ${ }^{19}$. Computational cost is a significant difficulty with equations of state based on fluid perturbation theory. W. Byers Brown ${ }^{20}$ developed an analytic representation of Kang et al.'s equation of state using Chebyshev polynomials. The accuracy of the above equations of state has been recently evaluated; those authors concluded that Ross's approach is the most reliable ${ }^{21}$. More recently, Fried and Howard have used a combination of integral equation theory and Monte Carlo simulations to generate a highly accurate equation of state for the exponential-6 fluid $^{22}$.

The exponential- 6 model is not well suited to molecules with a large dipole moment. Ree ${ }^{23}$ has used a temperature-dependent well depth $\varepsilon(\mathrm{T})$ in the exponential-6 potential to model polar fluids and fluid phase separations. Fried and Howard have developed an effective cluster model for $\mathrm{HF}^{24}$. The effective cluster model is valid to lower temperatures than the variable well-depth model, but it employs two more adjustable parameters. Jones et al ${ }^{25}$ have applied thermodynamic perturbation theory to polar detonation product molecules. More progress needs to be made in the treatment of polar detonation product molecules.

Efforts have been made to develop equations of state for detonation products based on direct Monte Carlo simulations instead of analytical approaches ${ }^{26,27}$. This 
approach is promising given recent increases in computational capabilities. One of the greatest advantages of direct simulation is the ability to go beyond van der Waals 1-fluid theory ${ }^{28}$.

In most cases, interactions between unlike molecules are treated with LorentzBerthelot combination rules ${ }^{29}$. These rules specify the interaction between unlike molecules as arithmetic or geometric averages of single molecule pair interactions. Nonadditive pair interactions have been used for $\mathrm{N}_{2}$ and $\mathrm{O}_{2}{ }^{18}$. The resulting $\mathrm{N}_{2}$ model accurately matches double shock data, but is not accurate at lower temperatures and densities $^{22}$. A combination of experiments on mixtures and theoretical developments is needed to develop reliable unlike-pair interaction potentials.

Many materials produce large quantities of solid products upon detonation. The most common solid detonation product is carbon, although some explosives produce aluminum and aluminum oxide ${ }^{30}$. Uncertainties in the equation of state and phase diagram of carbon remain a major issue in the thermochemical modeling of detonation. van Thiel and Ree have proposed an accurate Mie-Gruneisen equation of state for carbon ${ }^{31}$. Fried and Howard ${ }^{32}$ have developed a simple modified Murnaghan equation of state for carbon that matches recent experimental data on the melting line of graphite. Shaw and Johnson have derived a model for carbon clustering in detonation ${ }^{33}$. Viecelli and Ree have derived a carbon-clustering model for use in hydrodynamic calculations ${ }^{34,35}$.

The exp- 6 potential has also proved successful in modeling chemical equilibrium at the high pressures and temperatures characteristic of detonation. However, in order to calibrate the parameters for such models, it is necessary to have experimental data for molecules and mixtures of molecular species at high temperature and pressure. Static 
compression data, as well as sound speed measurements, provide important data for these models.

Exp-6 potential models can be validated through several independent means. Fried and Howard have considered the shock Hugoniots of liquids and solids in the "decomposition regime" where thermochemical equilibrium is established ${ }^{24,36}$. As an example of a typical thermochemical implementation, we consider the Cheetah thermochemical code. Cheetah is used to predict detonation performance for solid and liquid explosives. Cheetah solves thermodynamic equations between product species to find chemical equilibrium for a given pressure and temperature. From these properties and elementary detonation theory the detonation velocity and other performance indicators are computed.

Thermodynamic equilibrium is found by balancing chemical potentials, where the chemical potentials of condensed species are just functions of pressure and temperature, while the potentials of gaseous species also depend on concentrations. In order to solve for the chemical potentials, it is necessary to know the pressure-volume relations for species that are important products in detonation. Moreover, it is necessary to know these relations at the high pressures and temperatures that typically characterize the CJ state. Thus, there is a need for improved high-pressure equations of state for fluids, particularly for molecular fluid mixtures.

In addition to the exponential-6 potential, there is an intramolecular portion of the Helmholtz free energy. Cheetah uses a polyatomic model including electronic, 
vibrational, and rotational states. Such a model can be conveniently expressed in terms of the heat of formation, standard entropy, and constant pressure heat capacity of each species.

The modeling of high-pressure fluorocarbons is a good example of the equation of state modeling based on a simple exp-6 model. Fluorocarbons are challenging, due to the highly polar and associated nature of $\mathrm{HF}$, as well as the scarcity of experimental data on the equation of state of fluorinated compounds. A reactive fluorocarbon model requires parameters for hydrocarbons, fluorocarbon, $\mathrm{F}_{2}$, and HF. We determined hydrocarbon parameters with data from shock and static experiments. To our knowledge, highpressure equation of state measurements have not been performed on $F_{2}$. We use exp-6 parameters for $\mathrm{F}_{2}$ estimated by Zerilli and Jones ${ }^{37}$. We considered the shock behavior of polytetrafluoroethylene (PTFE) in order to determine parameters for $\mathrm{CF}_{4}$.

We predict that PTFE decomposes into a fluid phase composed mostly of $\mathrm{CF}_{4}$, and carbon in the diamond phase. We found an unusually high value of the potential steepness parameter $\alpha$. We note that Zerilli and Jones ${ }^{37}$ also obtained a very steep potential for $\mathrm{CF}_{4}$ by spherically averaging a Lennard-Jones potential.

The traditional exp-6 model has difficulty treating strongly associated fluids. HF is known to strongly associate ${ }^{38}$, in the gaseous, liquid, and fluid phases. Fried and Howard $^{24}$ have determined a simple HF association model that matches both high and low pressure data. The motivation for the cluster model is to determine the simplest possible model that will match both the low-pressure static compression of supercritical HF, and the shock Hugoniot of polyvinylidene fluoride (PVF2). The current model 
succeeds in this regard, although we cannot claim that the description of individual cluster species is quantitatively accurate. We have found that it is possible to match the shock Hugoniot of PVF2 without the association model, but the static compression requires an explicit treatment of association. We show the calculated isotherms of $\mathrm{HF}$ in Figure 3. The calculated shock Hugoniot of PVF2 is shown in Figure 4. In our calculations PVF2 dissociates to HF and carbon. The HF model is seen to work equally well in the experimental pressure range of 0.01 to $75 \mathrm{GPa}$.

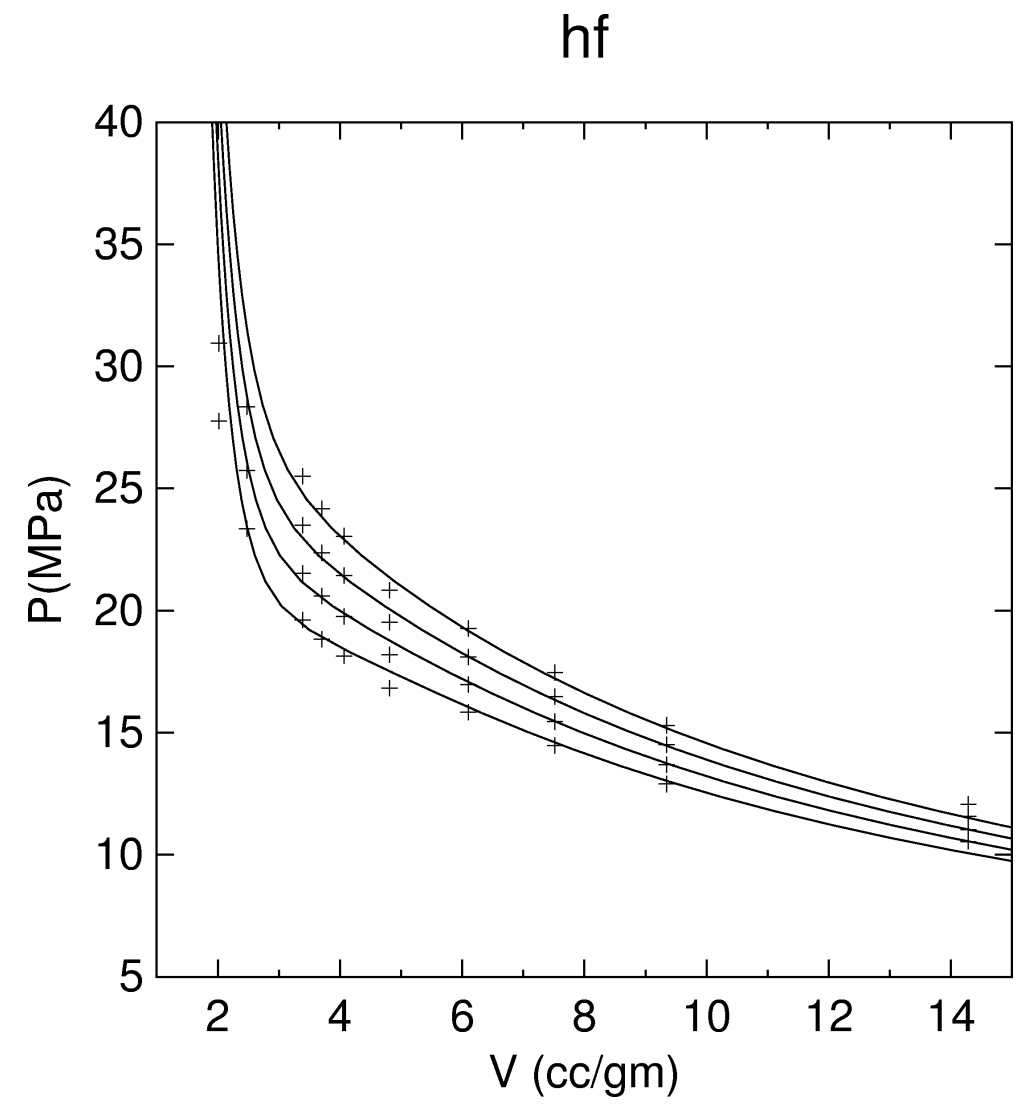

Figure 3: Measurements of the isotherms of $\mathrm{HF}$ at 543, 553, 563, and 573K (points) are compared to calculations of the present model (lines) 


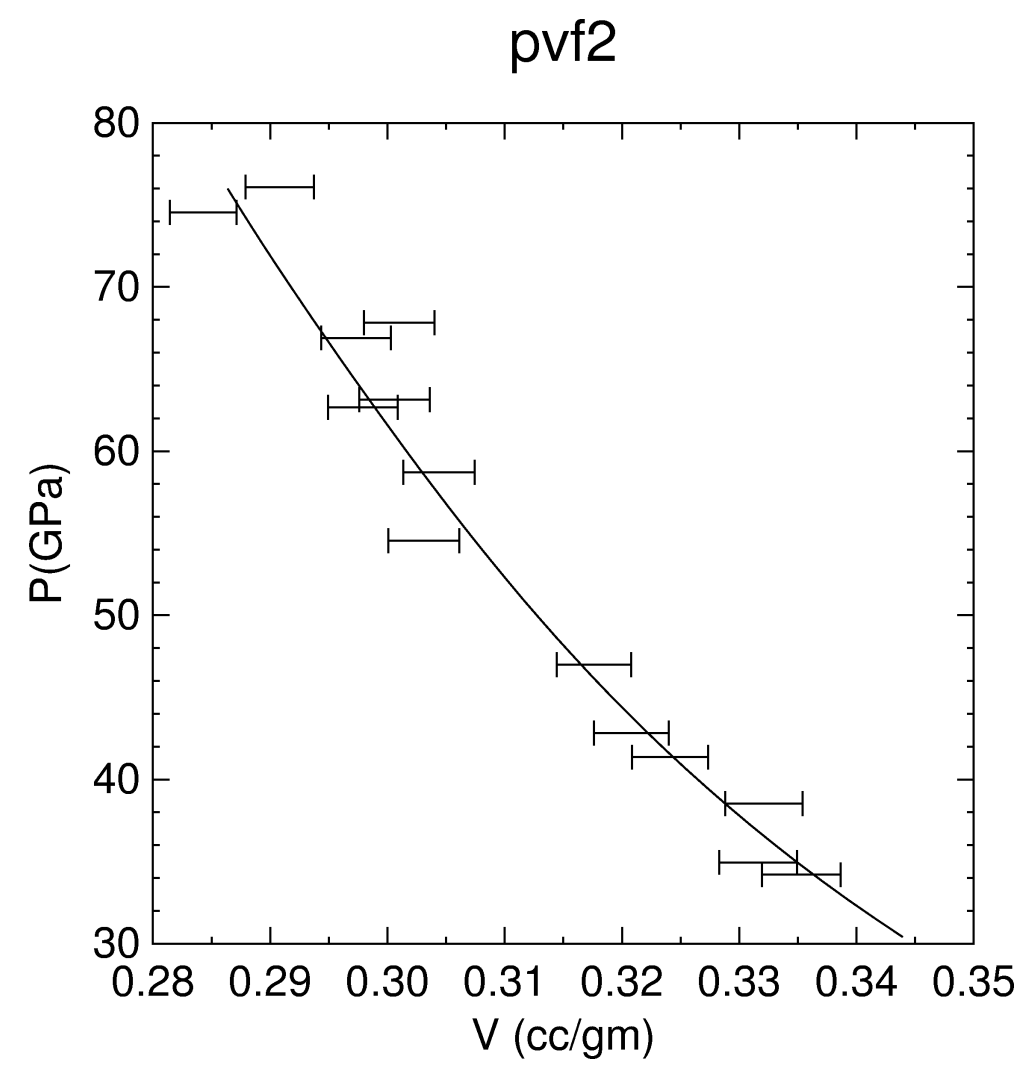

Figure 4. Measurements of the shock Hugoniot of PVF2 (error bars) are compared to calculations of the present model (line)

We now consider how the equation of state described above predicts the detonation behavior of condensed explosives. The overdriven shock Hugoniot of an explosive is an appropriate equation of state test. These states lie on the shock Hugoniot at pressures above the Chapman-Jouget point (see Figure 2). The overdriven Hugoniot tests the EOS across a broad range of thermodynamic states. The Hugoniot of PETN 
(penta-erythritol tetranitrate) is shown in Figure 5. We have calculated the Hugoniot with the EXP6 model presented here and also with the JCZS ${ }^{39}$ product library.

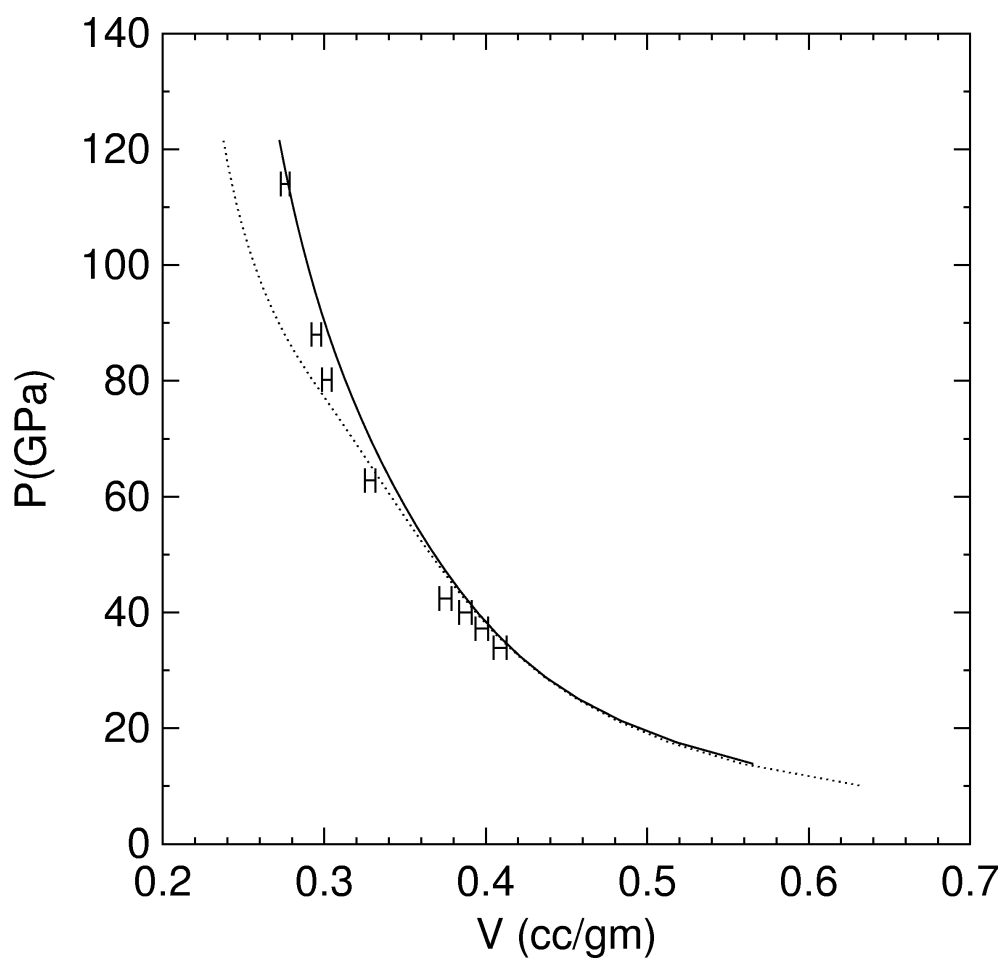

Figure 5: The shock Hugoniot of PETN as calculated with EXP6 (solid line) and the JCZS library (dotted line) vs. experiment (error bars).

Despite the many successes in the thermochemical modeling of energetic materials, there are several significant limitations. One limitation is that real systems do not always obtain chemical equilibrium during the relatively short (ns- $\mu \mathrm{s})$ timescales of detonation. When this occurs, quantities such as the energy of detonation and the detonation velocity are commonly over-predicted by a thermochemical calculation. 
Partial equilibrium calculations ${ }^{40}$ can overcome this problem. In partial equilibrium modeling, the concentrations of certain detonation products or reactants are held at fixed values. This assumes a priori knowledge of the timescales of reaction and detonation. Since this information is not usually known, partial equilibrium modeling is of limited predictive utility.

Chemical kinetic modeling is another possibility. There are several welldeveloped chemical kinetic mechanisms for highly studied materials such as RDX (cyclotrimethylene trinitramine) and $\operatorname{HMX}\left(1,3,5,7\right.$-tetranitro-1,3,5,7-tetraazaoctane) ${ }^{41,42}$ - Unfortunately, detailed chemical kinetic mechanisms are not available for highpressure conditions. Some workers have applied simplified chemical kinetics to detonation processes ${ }^{43}$. The primary difficulty in high-pressure chemical kinetic models is a lack of experimental data on speciation. First principles simulations, discussed below, have the potential to provide chemical kinetic information on fast processes. This information could then conceivably be applied to longer timescales and lower temperatures using high-pressure chemical kinetics.

Finally, there are several issues to be addressed in determining the equation of state of detonation products. While convenient, the exponential-6 fluid does not adequately treat electrostatic interactions. In the condensed phase, effects such as dielectric screening and charge-induced dipoles need to be considered. Molecular shape is also neglected in exponential-6 modeling. While the small size of most detonation product molecules limits the importance of molecular shape, lower temperature conditions could yield long chain molecules. Also, ionization may become dominant at 
high temperatures or for systems with strong acids and bases. Equation of state information for molecular ions and a successful statistical mechanical treatment at high pressure do not yet exist.

\section{ATOMISTIC MODELING OF CONDENSED-PHASE REACTIONS}

Chemical equilibrium methods provide useful predictions of macroscopic detonation processes and corresponding resultant products. However, no details on the atomistic mechanisms in the detonation process are revealed. We now discuss condensedphase detonation simulations using atomistic modeling techniques. Such simulations are quite useful for understanding the condensed-phase reaction mechanisms that can occur on the microscopic level.

Numerous experimental studies have investigated the atomistic details of HE decomposition by examining the net products after thermal (low pressure) decomposition. More specifically for RDX and HMX, there exists a solid understanding of the putative rate limiting reaction $\left(\mathrm{NO}_{2}\right.$ dissociation $)$ and the final products of the decomposition process (for references see other chapters herein). Several theoretical studies have also been reported on the energetics of gas-phase decomposition pathways for HE materials using a variety of methods. For example, we point to work in RDX and HMX where both quantum chemistry ${ }^{44-47}$ and classical simulations of unimolecular dissociation ${ }^{48,49}$ were used.

The gas-phase results provide much insight into the reaction pathways for isolated HE molecules; however, the absence of the condensed-phase environment is known to strongly affect reaction pathways. Some of the key questions related to condensed-phase decomposition are 1). How do the temperature and pressure affect the reaction pathways? 
2). Are there temperature or pressure-induced phase-transitions which play a role in the reaction pathways that may occur? 3). What happens to the reaction profiles in a shockinduced detonation? These questions can only be answered with condensed-phase simulations, but would require large-scale reactive chemical systems (1000s of atoms). Here we present very recent results of condensed-phase atomistic simulations, which are pushing the envelope towards reaching the required simulation goal.

\section{Molecular-Dynamics with Bond-Order Potentials}

A novel approach for investigating reactions in the condensed-phase is to take advantage of the computational efficiency of empirical force. Although traditional empirical force fields cannot model bond-breaking events, additional force field terms dependent on the bond-order can be included to model bond breaking. These bond-order terms then "semi-empirically" describe the bond-breaking mechanisms needed for simulating reaction conditions. The bond order defines the strength of the bond between two atoms where larger numbers imply stronger bonds; it is a measure of the net number of bonds between a specific pair of atoms in a molecule. Bond-order potentials have been proposed early in the history of atomistic modeling; several methods exist, and each has a different prescription for defining the bond order between reactants and products. ${ }^{50-52}$ Recently Goddard et al. have perfected their own method (called ReaxFF) for hydrocarbons and energetic materials (more specifically, RDX). ${ }^{53}$

In the ReaxFF method, the central force formalism for describing force fields is still used, but non-bonded interactions and Coulomb forces are included to yield smooth bond dissociations between atoms. Local perturbations (bond, angle, and torsion, etc.) are 
also added to describe complex molecules more accurately. The bond order term, $\mathrm{BO}_{\mathrm{ij}}{ }^{\prime}$ between a pair of atoms is of the form

$$
B O_{i j}^{\prime}=\exp \left[p_{1 \sigma}\left(\frac{r_{i j}}{r}\right)^{p_{2 \sigma}}\right]+\exp \left[p_{1 \pi}\left(\frac{r_{i j}}{r}\right)^{p_{2 \pi}}\right]+\exp \left[p_{1 \pi^{\prime}}\left(\frac{r_{i j}}{r}\right)^{p_{2 \pi^{\prime}}}\right]
$$

The parameters, $\mathrm{p}$, correspond to the bond order curves associated with different types of orbitals for each atom type (see Fig. 6 for the C-C case). The method is shown to yield heats of formations that are within 1-2 kcal/mol compared with experimental values. In addition, the energetics of dissociations yield the proper qualitative features compared to quantum chemistry calculations.

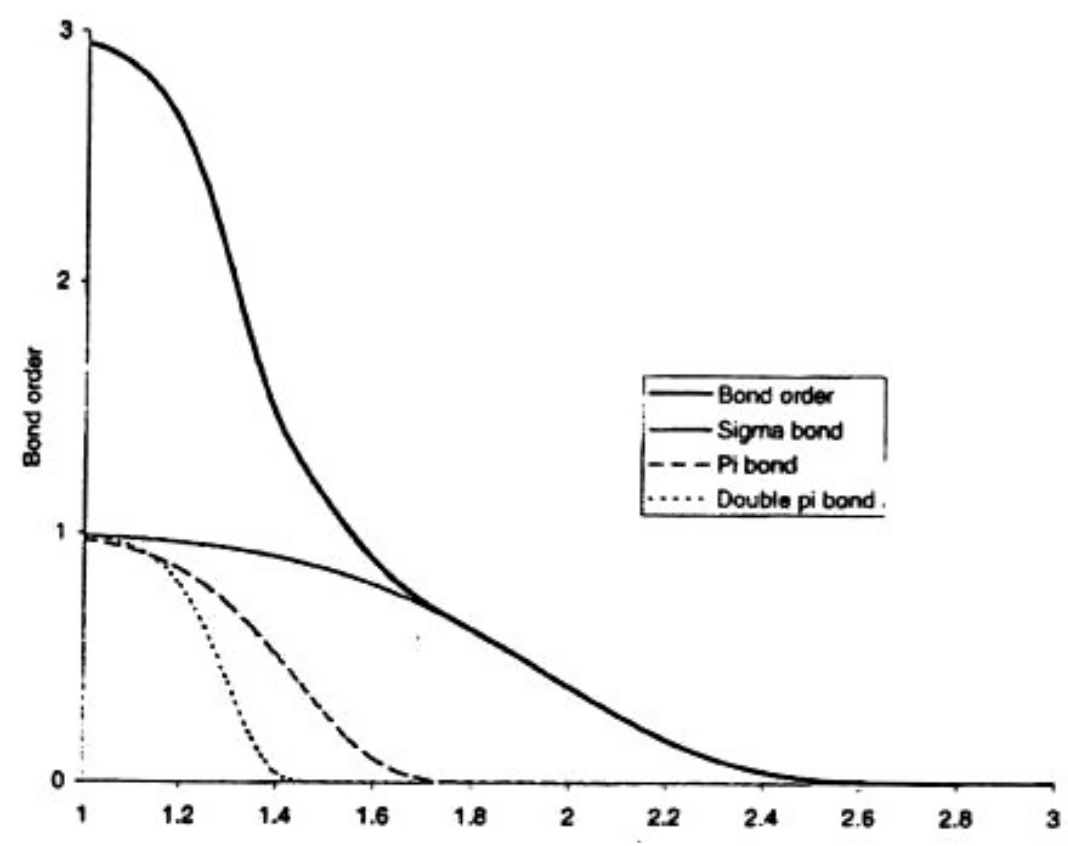

Figure 6: Interatomic distance dependency of the carbon-carbon bond order.

Recent results where ReaxFF was applied to RDX demonstrate the method's capabilities. Strachan et al. applied the ReaxFF method to investigate the initial stages of a shock-induced detonation of RDX. Parameters were developed from 40 different 
reactions and 1600 different equilibrated molecules, which represent potential product molecules along the possible reaction pathways. Two two-dimensional periodic slabs (each slab having $32 \mathrm{RDX}$ molecules forming a perfect crystal) were impacted into each other. Impact velocities were chosen at 2, 4, 6, 8, and $10 \mathrm{~km} / \mathrm{s}$.

The results of these shock-induced detonation simulations are quite interesting. First, the products yielded from the simulations (shown in Fig. 7) are products that are observed experimentally. ${ }^{54,55}$ Second, the primary reactions leading to $\mathrm{NO}_{2}, \mathrm{OH}, \mathrm{NO}$, and $\mathrm{N}_{2}$ occur at very early stages of the simulations. Additionally, as the impact velocity increases, $\mathrm{N}_{2}$ and $\mathrm{OH}$ become the dominant product species at short times. Finally, the simulations show that although the barrier for the pathways leading to $\mathrm{NO}_{2}$ and $\mathrm{HONO}$ is essentially the same, $\mathrm{NO}_{2}$ is the main product for low shock velocities $(<6 \mathrm{~km} / \mathrm{s})$, in agreement with experimental work by Owens and Sharma. ${ }^{56}$

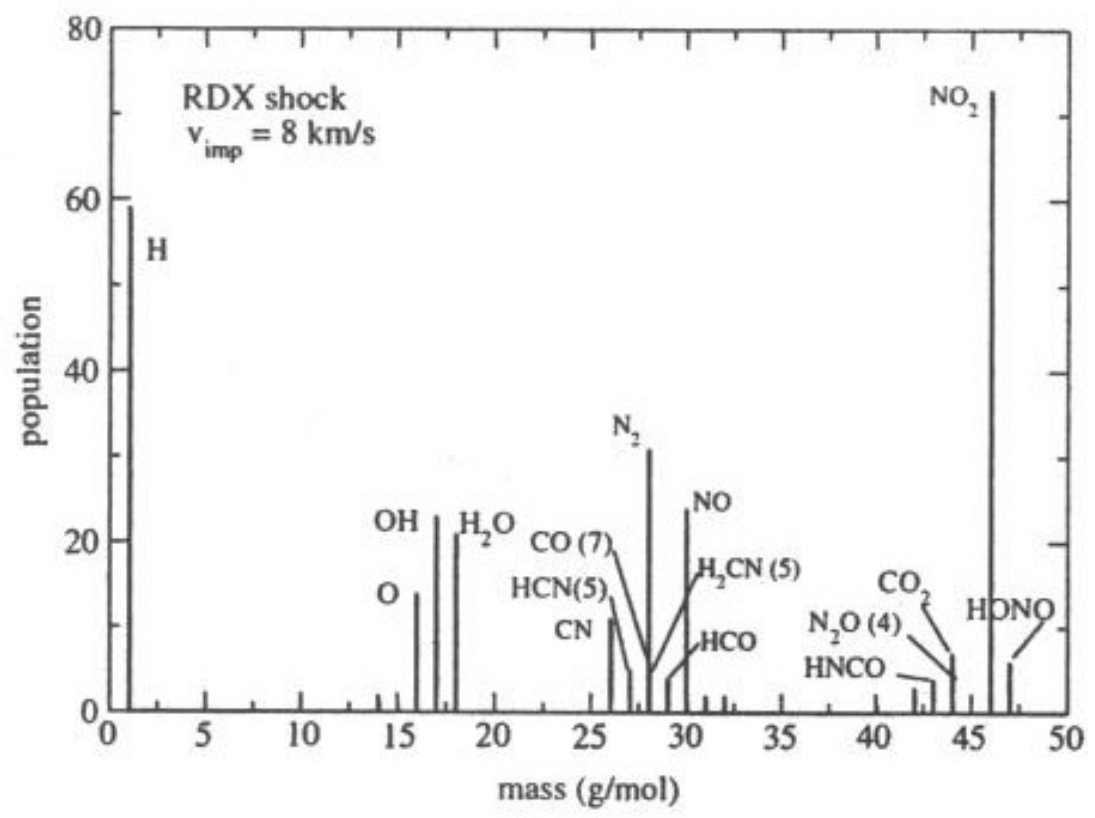


Figure 7. Mass spectrum corresponding to $v_{i m p}=8 \mathrm{~km} / \mathrm{s}$ at time $\mathrm{t}=4 \mathrm{ps}$. Population as a function of mass for all the molecules found up to mass $50 \mathrm{~g} / \mathrm{mol}$ (all species with population larger than 3 are labeled).

\section{Molecular-Dynamics with Quantum Mechanical Methods}

Bond-order potentials are fast and appear to give proper qualitative results; however, there are difficulties in using such methods. First, many parameters must be added to model the correct reaction pathway. Second, many quantum chemistry calculations of small systems must be done to fit these parameters. For systems like RDX and HMX, which are experimentally well-defined, in terms of known products and initial reaction pathways, bond-order potentials work very well and yield greater understanding of these systems. For less known systems, for example TATB $(1,3,5-$ triamino-2,4,6-trinitrobenzene), methods using bond-order potentials may be less accurate.

Recently, quantum mechanical methods have been applied to systems with up to 1,000 atoms. This is due not only to speed-ups in computer technology, but also improvements in algorithms. A wide range of approximations can also be made to yield a variety of methods; each able to address a different range of questions based on the accuracy of the method chosen. We now discuss a range of quantum mechanical based methods used to answer specific questions regarding shock-induced detonation conditions. 
Atomistic simulations have recently been performed on condensed-phase HMX (1,3,5, 7-tetranitro-1, 3,5,7-tetraazacyclooctane). This material is widely used as an ingredient in various explosives and propellants. A molecular solid at standard state, it has four known polymorphs, one of which, the $\delta$ phase is comprised of six molecules per unit cell. In a recent study, Manaa et al. have conducted a quantum-based molecular dynamics simulation of the chemistry of HMX under extreme conditions, similar to those encountered at the Chapman-Jouget detonation state ${ }^{57}$. The simulation studied the reactivity of dense $\left(1.9 \mathrm{~g} / \mathrm{cm}^{3}\right)$ fluid $\mathrm{HMX}$ at $3500 \mathrm{~K}$ for extended reaction times of up to $55 \mathrm{ps}$, thus allowing the formation of stable product molecules.

There are numerous experimental characterizations at low temperatures (i.e. $<$ $1000 \mathrm{~K}$, well below detonation temperature) of the decomposition products of condensedphase HMX. ${ }^{58-68}$ These studies tend to identify final gas products (such as $\mathrm{H}_{2} \mathrm{O}, \mathrm{N}_{2}, \mathrm{H}_{2}$, $\mathrm{CO}, \mathrm{CO}_{2}$, etc.) from the surface burn, and aspire to establish a global decomposition mechanism.

To date, experimental work on thermal decomposition of condensed phase HMX is largely restricted to relatively low temperature $(\sim 550 \mathrm{~K})$ and pressure $(0.1 \mathrm{GPa})$ regimes. Similar experimental observations at detonation conditions (temperatures 2000$5000 \mathrm{~K}$, and pressure 10-30 GPa), however, have not been realized to date. Computer simulations provide the best access to the short time scale processes occurring in these regions of extreme conditions of pressure and temperature. ${ }^{69}$ In particular, simulations employing many-body potentials, ${ }^{70-72}$ or tight-binding based methods have emerged as viable computational tools, the latter has been successfully demonstrated in the studies of shocked hydrocarbons. ${ }^{73,74}$ 
Lewis et al. ${ }^{75}$ calculated four possible decomposition pathways of the $\alpha$-HMX polymorph: $\mathrm{N}_{-} \mathrm{NO}_{2}$ bond dissociation, $\mathrm{HONO}$ elimination, $\mathrm{C}-\mathrm{N}$ bond scission, and the concerted ring fission. Based on the energetics, it was determined that $\mathrm{N}-\mathrm{NO}_{2}$ dissociation was the initial mechanism of decomposition in the gas phase, while they proposed $\mathrm{HONO}$ elimination and $\mathrm{C}-\mathrm{N}$ bond scission to be favorable in the condensed phase. The more recent study of Chakraborty et al. ${ }^{42}$, using the DFT(B3LYP) method, reported detailed decomposition pathways of the $\beta$-HMX, the stable polymorph at room temperature. It was concluded that consecutive HONO elimination (4HONO) and subsequent decomposition into $\mathrm{HCN}, \mathrm{OH}$ and $\mathrm{NO}$ are energetically the most favorable pathways in the gas phase. The results also showed that the formation of $\mathrm{CH}_{2} \mathrm{O}$ and $\mathrm{N}_{2} \mathrm{O}$ could occur preferably from secondary decomposition of methylenenitramine. While these studies concentrated on gas phase decomposition mechanisms, to date no computational treatment of condensed phase reaction mechanisms exist. Other theoretical studies were concerned with the derivation of a force field from first principle calculations, ${ }^{76}$ and the application of classical molecular dynamics as in simulating pressure effects on crystal packing. ${ }^{77,78}$

The computational approach employed by Manaa et al. ${ }^{57}$ to simulate the condensed-phase chemical reactivity of HMX is based on implementing the selfconsistent charge density-functional tight binding (SCC-DFTB) scheme. ${ }^{79}$ This is an extension of the standard tight binding approach in the context of density functional theory that describes total energies, atomic forces, and charge transfer in a self-consistent manner. The initial condition of the simulation included six HMX molecules in a cell, corresponding to the unit cell of the $\delta$ phase of HMX with a total of 168 atoms. 
The density of $1.9 \mathrm{~g} / \mathrm{cm}^{3}$ and a temperature of $3500 \mathrm{~K}$ were chosen. This state is in the neighborhood of the Chapman-Jouget state of $\beta$-HMX $\left(3500 \mathrm{~K}, 2.1 \mathrm{~g} / \mathrm{cm}^{3}\right)$ as predicted through thermochemical calculations described later. The closest experimental condition corresponding to this simulation would be a sample of HMX, which is suddenly heated under constant volume conditions, such as in a diamond anvil cell. The molecular dynamics simulation was conducted at constant volume and constant temperature. Periodic boundary conditions, whereby a particle exiting the cell on one side is reintroduced on the opposing side with the same velocity were imposed.

Under the simulation conditions, the HMX was in a highly reactive dense fluid phase. There are important differences between the dense fluid (supercritical) phase and the solid phase, which is stable at standard conditions. Namely, the dense fluid phase cannot accommodate long-lived voids, bubbles, or other static defects. Instead numerous fluctuations in the local environment occur within a timescale of 10 s of femtoseconds (fs). The fast reactivity of the dense fluid phase and the short spatial coherence length make it well suited for molecular dynamics study with a finite system for a limited period of time. Under the simulation conditions chemical reactions occurred within $50 \mathrm{fs}$. Stable molecular species were formed in less than one ps. Fig. 8 displays the product formation of $\mathrm{H}_{2} \mathrm{O}, \mathrm{N}_{2}, \mathrm{CO}_{2}$ and $\mathrm{CO}$, respectively. The concentration, $\mathrm{C}(t)$, is represented by the actual number of product molecules formed at the corresponding time $t$. Each point on the graphs (open circles) represents a $250 \mathrm{fs}$ averaged interval. The number of the molecules in the simulation was sufficient to capture clear trends in the chemical composition of the species studied. It is not surprising that the rate of $\mathrm{H}_{2} \mathrm{O}$ formation is much faster than that of $\mathrm{N}_{2}$. Fewer reaction steps are required to produce a triatomic 
species like water, while the formation of $\mathrm{N}_{2}$ involves a much more complicated mechanism. ${ }^{41}$ Further, the formation of water starts around $0.5 \mathrm{ps}$ and seems to have reached a steady state at $10 \mathrm{ps}$, with oscillatory behavior of decomposition and formation clearly visible. The formation of $\mathrm{N}_{2}$, on the other hand, starts around $1.5 \mathrm{ps}$ and is still progressing (slope of the graph is slightly positive) after $55 \mathrm{ps}$ of simulation time, albeit at small variation.
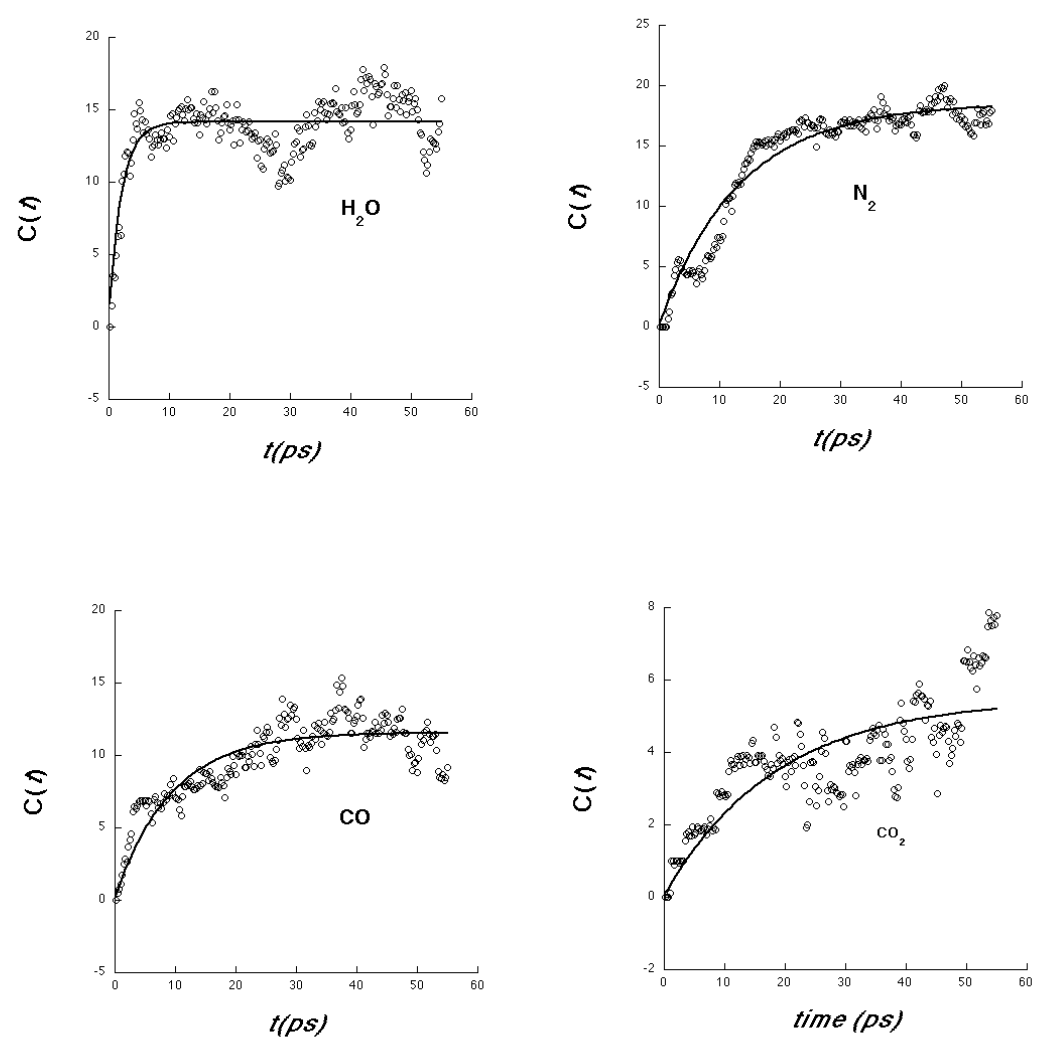

Fig. 8. Product particle-number formations as a function of time of $\mathrm{H}_{2} \mathrm{O}, \mathrm{N}_{2}, \mathrm{CO}_{2}$, and CO. 
Due to the lack of high-pressure experimental reaction rate data for this (and other) explosive(s) with which to compare with, we produce in Fig.9 a comparison of dominant species formation for decomposing HMX obtained from entirely different theoretical approach. The concentration of species at chemical equilibrium can be estimated through thermodynamic calculations, as implemented within the Cheetah thermochemical code. ${ }^{22,32}$

As can be noticed in Fig.9, the results of the MD simulation compare very well with the formation of $\mathrm{H}_{2} \mathrm{O}, \mathrm{N}_{2}$, and $\mathrm{HNCO}$. The relative concentration of $\mathrm{CO}$ and $\mathrm{CO}_{2}$, however, is reversed at the limited time of the simulation. In addition, Cheetah predicted that carbon in the diamond phase was in equilibrium with the other species at a concentration of $4.9 \mathrm{~mol} / \mathrm{kg}$ HMX. No condensed carbon was found in the current simulation. Several other products and intermediates with lower concentrations, common to the two methods, have also been identified. These include $\mathrm{HCN}, \mathrm{NH}_{3}, \mathrm{~N}_{2} \mathrm{O}, \mathrm{CH}_{3} \mathrm{OH}$, and $\mathrm{CH}_{2} \mathrm{O}$. It is hoped that interplay between the two vastly different approaches could be established at much longer simulation time. The goal will be to expand the product molecule set of the thermochemical code with important species determined from our ab initio based simulations for kinetic modeling. 


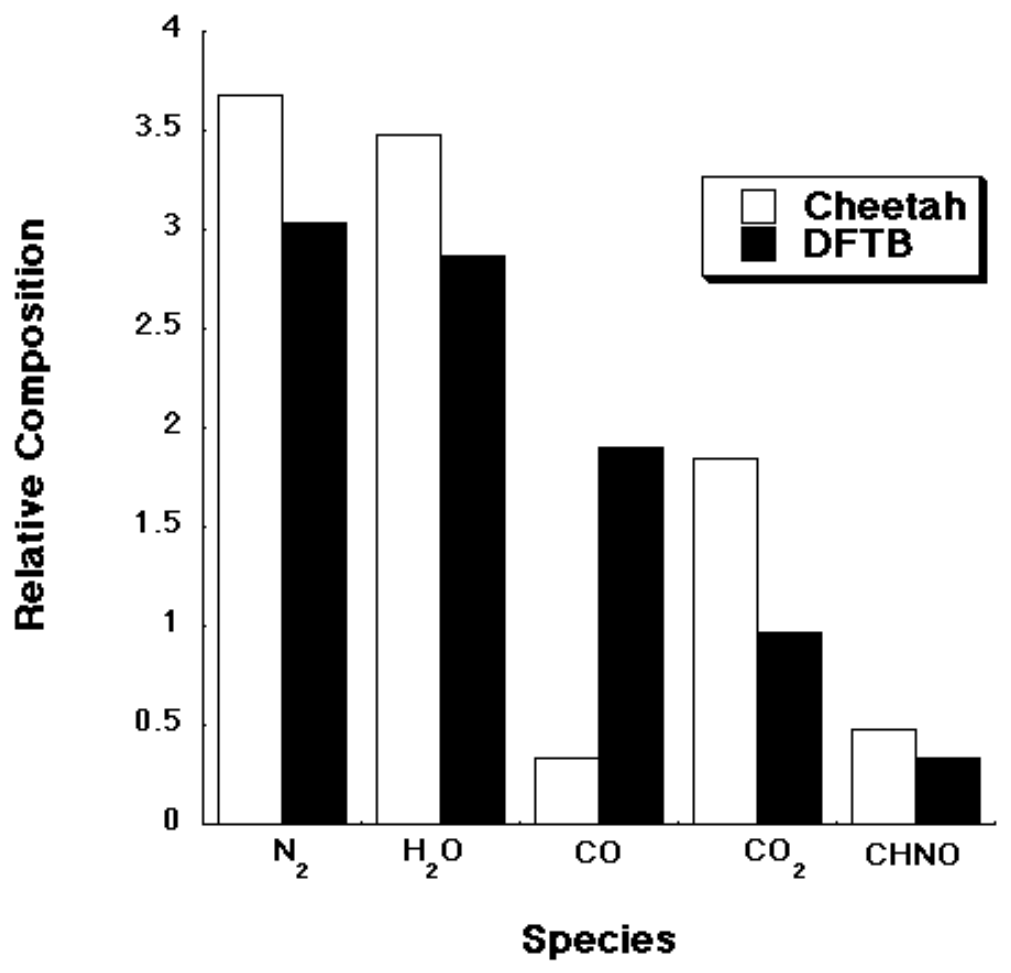

Fig.9. Comparison of relative composition of dominant species determined from current DFTB simulation and from a thermodynamical calculation.

One expects more $\mathrm{CO}_{2}$ than $\mathrm{CO}$ as final products, as predicted by Cheetah (Fig.9). The results displayed in Figs. 8 show that, at simulation time of 40 ps, the system is still in the second stage of reaction chemistry. At this stage, the $\mathrm{CO}$ concentration is rising and has not yet undergone the water gas shift reaction $\left(\mathrm{CO}+\mathrm{H}_{2} \mathrm{O} \rightarrow \mathrm{CO}_{2}+\mathrm{H}_{2}\right)$ conversion. Interestingly, this shift seems to occur at around $50 \mathrm{ps}$ of the simulation, with $\mathrm{CO}_{2}$ molecules are being formed while $\mathrm{CO}$ concentration is correspondingly diminishing.

Although the simulation sheds light on the chemistry of HMX under extreme conditions, there are methodological shortcomings that need to be overcome in the future. The demanding computational requirements of the present method limits the method's 
applicability to short times and corresponding high temperature conditions. A second issue is that the SCC-DFTB method is not as accurate as more elaborate ab initio methods. Nonetheless, the present approach could still be considered as a promising direction for future research on the chemistry of energetic materials.

\section{QUANTIFYING THE ENERGETICS OF REACTION PATHWAYS}

The energetics of particular reactions pathways, once known, can be more accurately quantified than is possible in a calculation based on tight binding methods or classical potentials. For instance, density functional calculations can be undertaken with

large basis sets ${ }^{80,81}$. It is also possible to investigate the importance of excited electronic states using sophisticated electron correlation techniques ${ }^{82,83}$.

One of the more prevalent products of HMX decomposition is HONO. Formation of HONO can likely occur from unimolecular dissociation where the process of this reaction is an hydrogen transfer from the $\mathrm{CH}_{2}$ group to the $\mathrm{NO}_{2}$ group (see Scheme I). ${ }^{84}$ However, the molecular packing in HMX would seem to suggest that hydrogen transfer could occur between adjacent molecules; there is a very weak hydrogen-bonding-like interaction from the $\mathrm{C}-\mathrm{H}$ of the $\mathrm{CH}_{2}$ group of one molecule to the $\mathrm{O}$ of the $\mathrm{NO}_{2}$ group of its adjacent molecule. 


\section{Scheme I}

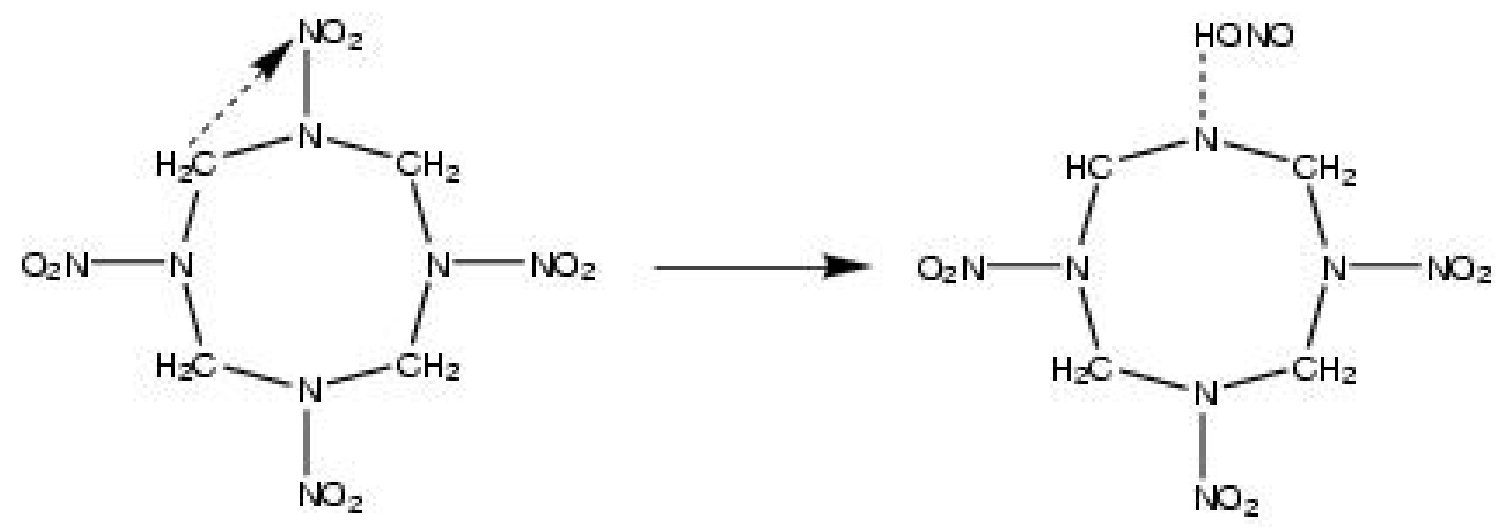

Previous work by Lewis investigated the energetics of HONO formation in condensed-phase HMX, where intermolecular hydrogen transfer occurs. Results on the energetics of HONO formation in the three pure polymorphic phases $(\alpha, \beta$, and $\delta)$ are presented here using the $a b$ initio tight-binding method called FIREBALL. This method was also used previously the energetics of the three pure polymorphic HMX phases. ${ }^{75}$

\section{ELECTRONIC EXCITATIONS IN SHOCK-INDUCED EXPLOSIVES}

All calculations discussed above assume that excited electronic states do not significantly contribute to the chemistry of detonating energetic materials. In 1971, Williams suggested that excited electronic states play a role in the initiation and propagation of detonation waves in explosives. ${ }^{85}$ Kuklja et al. demonstrated a practical use of quantum mechanical methods, in particular periodic Hartree-Fock calculations. ${ }^{86}$ They investigated the electronic states in RDX with edge dislocation defects included in the simulation cell. It is proposed that such defects provide local sites for 'hot spots', which localize the energy of the impact wave and trigger chemical reactions. Kuklja 
proposed that the edge dislocation defects in RDX introduce states in the gap; therefore, these localized defect states more readily produce electronic excitations or 'hot spot' regions. The effect becomes more pronounced under higher pressures as the gap between the occupied and unoccupied states decreases, which implies that the excitations become more energetically favorable.

In other work, Reed et al. consider bandgap lowering under uniaxial strain due to molecular defects and vacancies. ${ }^{80}$ The method they use is an ab initio molecular dynamics method using density-functional theory with gradient corrected exchangecorrelation functionals (PW91 and PBE). Pseudopotentials were used with a plane wave basis set. Simulations of all possible nearest-neighbor collisions at a shock front indicate that there is a bandgap lowering effect due to the molecular defects and vacancies. However, the bandgap is not lowered enough to produce a significant population of excited states in the crystal. Furthermore, dynamical effects have a more significant effect on the bandgap than static effects, but relative molecule velocities in excess of $6 \mathrm{~km} / \mathrm{s}$ are required to produce a significant thermal population of excited states.

\section{CONCLUSIONS}

The ability to model chemical reaction processes in condensed phase energetic materials is rapidly progressing. Chemical equilibrium modeling is a mature technique with some limitations. Progress in this area continues, but is hampered by a lack of knowledge of condensed phase reaction mechanisms and rates. Atomistic modeling is much more computationally intensive, and is currently limited to very short time scales. Nonetheless, this methodology promises to yield the first reliable insights into the 
condensed phase processes responsible for high explosive detonation. Further work is necessary to extend the timescales involved in atomistic simulations. Recent work in implementing thermostat methods appropriate to shocks ${ }^{87,88}$ may promise to overcome some of these difficulties.

Most current work on energetic material reactivity assumes that electronically adiabatic processes dominate. The role of excited states is becoming clearer, however. These states are not accessible in perfect crystals under realistic pressures and temperatures, but may still be accessed through defects or other energy localization mechanisms.

\section{ACKNOWLEDGEMENTS}

This research work was performed under the auspices of the U.S. Department of Energy by the University of California, LLNL under contract number W-7405-Eng-48.

\section{REFERENCES}

(1) Bishop, R.; Harradine, D.; Flesner, R.; Larsen, S.; Bell, D. Ind. Eng. Chem. Res. 2000, 39, 1215.

(2) Hawthorne, S. B.; Lagadec, A. J. M.; Kalderis, D.; Lilke, A. V.; Miller, D. J. Environ. Sci. Technol. 2000, 34, 3224.

(3) Zel'dovich, Y. B.; Raiser, Y. P. Physics of Shockwaves and High Temperature Hydrodynamics Phenomena; Academic Press: New York, 1966.

(4) Fried, L. E.; Manaa, M. R.; Pagoria, P. F.; Simpson, R. L. Annu. Rev. Mater. Res. 2001, 31, 291.

(5) Souers, P. C.; Kury, J. W. Propellants, Explosives, Pyrotechnics 1993, 18, 175.

(6) Fickett, W.; Davis, W. C. Detonation; University of California Press: Berkeley, 1979.

(7) Kistiakowsky, G. B.; Wilson, E. B. "Report on the prediction of detonation velocities of solid explosives," Office of Scientific Research and Development, 1941.

(8) Finger, M.; Lee, E.; Helm, F. H.; Hayes, B.; Hornig, H.; McGuire, R.; Kahara, M.; Guidry, M. "The effect of elemental composition on the detonation behavior of explosives"; Sixth Symposium (International) on Detonation, 1976, Coronado, CA. 
(9) Mader, C. L. Numerical modeling of detonations; University of California Press: Berkeley, CA, 1979.

1152.

(10) Gubin, S. A.; Odintsov, V. V.; Pepekin, V. I. Sov. J. Chem. Phys. 1985, 3,

(11) Hobbs, M. L.; Baer, M. R. "Calibrating the BKW-EOS with a large product species data based and measured C-J properties"; Tenth International Detonation Symposium, 1993, Boston, MA.

(12) Fried, L. E.; Souers, P. C. Propellants, Explosives, Pyrotechnics 1996, 21, 215.

(13) Cowperthwaite, M.; Zwisler, W. H. "The JCZ equation of state for detonation products and their incorporation into the TIGER code"; Sixth Detonation Symposium, 1976.

(14) Ross, M. J. Chem. Phys. 1979, 71, 1567.

(15) Ross, M.; Ree, F. H. J. Chem. Phys. 1980, 73, 6146.

(16) Ree, F. H. J. Chem. Phys. 1984, 81, 1251.

(17) van Thiel, M.; Ree, F. H. J. Appl. Phys. 1987, 62, 1761.

(18) van Thiel, M.; Ree, F. H. J. Chem. Phys. 1996, 104, 5019.

(19) Kang, H. S.; Lee, C. S.; Ree, T.; H., R. F. J. Chem. Phys. 1985, 82, 414.

(20) Byers Brown, W. J. Chem. Phys. 1987, 87, 566.

(21) Charlet, F.; Turkel, M. L.; Danel, J. F.; Kazandjian, L. J. Appl. Phys.

1998, 84, 4227.

(22) Fried, L. E.; Howard, W. M. J. Chem. Phys. 1998, 109, 7338.

(23) Ree, F. H. J. Chem. Phys. 1986, 84, 5845.

(24) Fried, L. E.; Howard, W. M. J. Chem. Phys. 1999, 110, 12023.

(25) Jones, H. D. "Theoretical equation of state for water at high pressures";

Shock Compression of Condensed Matter, 2001, 2001, Atlanta, Georgia.

(26) Shaw, M. S. J. Chem. Phys. 1991, 94, 7550.

(27) Brennan, J. K.; Rice, B. M. Phys. Rev. E 2002, 66, 021105.

(28) Leland, T. W.; Rowlinson, J. S.; Sather, G. A. Trans. Faraday Soc. 1947, $64,1447$.

(29) Reed, T. M.; Gubbins, K. E. Statistical Mechanics; McGraw-Hill: New

York, 1973.

(30) Jones, H. D.; Zerilli, F. J. J. Appl. Phys. 1991, 69, 3893.

(31) van Thiel, M.; Ree, F. H. Int. J. of Thermophysics 1989, 10, 227.

(32) Fried, L. E.

(33) Shaw, M. S.; Johnson, J. D. J. Appl. Phys. 1987, 62, 2080.

(34) Viecelli, J. A.; Ree, F. H. J. Appl. Phys. 1999, 86, 237.

(35) Viecelli, J. A.; Ree, F. H. J. Appl. Phys. 2000, 88, 683.

(36) Fried, L. E.; Howard, W. M.; Souers, P. C. "EXP6: A new equation of state library for high pressure thermochemistry"; 12 Symposium (International) on Detonation, 2002, San Diego, CA.

(37) Zerilli, F. J.; Jones, H. D. "The equation of state of HF"; High pressure science and technology, 1993, New York, NY.

(38) Klein, M. L.; McDonald, I. R. J. Chem. Phys. 1979, 71, 298.

(39) Hobbs, M. L.; Baer, M. R.; McGee, B. C. Propellants, Explosives, Pyrotechnics 1999, 24, 269. 
(40) Cowperthwaite, M. "Nonideal detonation in a composite CHNO explosive containing aluminum"; Tenth International Detonation Symposium, 1993, Boston, Massachusetts.

(41) Melius, C. F. HMX decomposition. In Chemistry and Physics of Energetic Materials; Bulusu, D. N., Ed.; Kluwer: Dordercht, 1990.

(42) Chakraborty, D.; Muller, R. P.; Dasgupta, S.; Goddard III, W. A. J. Phys. Chem. A 2001, 105, 1302.

(43) Howard, W. M.; Fried, L. E.; Souers, P. C. "Kinetic Modeling of nonideal explosives with Cheetah"; 11th International Symposium on Detonation, 1998, Snowmass, CO.

(44) Wu, C.; Fried, L. J. Phys. Chem. A 1997, 101, 8675.

(45) Zhang, S.; Truong, T. J. Phys. Chem. A 2001, 105, 2427.

(46) Lewis, J.; Glaesemann, K.; VanOpdorp, K.; Voth, G. 2001.

(47) Chakraborty, D.; Muller, R.; Dasgupta, S.; W.A. Goddard, I. J. Phys. Chem. A 2001, 105, 1302.

(48) Sewell, T.; Thompson, D. J. Phys. Chem. 1991, 95, 6228.

(49) Chambers, C.; Thompson, D. J. Phys. Chem. 1995, 99, 15881.

(50) Johnston, H. S. Adv. Chem. Phys. 1960, 3, 131.

(51) Johnston, H. S.; Parr, C. J. Am. Chem. Soc. 1963, 85, 2544.

(52) Landis, C. M.; Cleveland, T.; Firman, T. K. J. Am. Chem. Soc. 1998, 120, 2641.

(53) Duin, A. T. C. v.; Dasgupta, S.; Lorant, F.; W.A. Goddard, I. J. Phys.

Chem. 2001, 105, 9396.

(54) Behrens, R. J. Phys. Chem. 1990, 94, 6706.

(55) Behrens, R. J. Phys. Chem. 1991, 95, 5838.

(56) Owens, F. J.; Sharma, J. J. Appl. Phys. 1979, 51, 1494.

(57) Manaa, M. R.; Fried, L. E.; Melius, C. F.; Elstner, M.; Frauenheim, T. J. Phys. Chem. A 2002, 106, 9024.

(58) Suryanarayana, B.; Graybush, R. J.; Autera, J. R. Chem. Ind. London 1967, 52, 2177.

13.

(59) Bulusu, S.; Axenrod, T.; Milne, G. W. A. Org. Mass. Spectrom. 1970, 3 , 1979.

(60) Farber, M.; Srivastava, R. D. "HMX3"; 16th JANNA Combus. Meeting,

(61) Morgan, C. V.; Bayer, R. A. Combust. Flame 1979, 36, 99.

(62) Fifer, R. A. Fundamentals of Solid Propellant Combustion. In Progress in Astronautics and Aeronautics; Kuo, K. K., Summerfield, M., Eds.; AIAA Inc.: New York, 1984; Vol. 90; pp 177.

(63) Behrens, R. Int. J. Chem. Kinet. 1990, 22, 135.

(64) Behrens, R.; Bulusu, S. J. Phys. Chem. 1991, 95, 5838.

(65) Oxley, J. C.; Kooh, A. B.; Szekers, R.; Zhang, W. J. Phys. Chem. 1994, $98,7004$.

(66) Brill, T.; Gongwer, P.; Williams, G. J. Phys. Chem. 1994, 98, 12242.

(67) Brill, T. B. J. Prop. Power 1995, 11, 740. 117,170 .

(68) Tang, C.-J.; Lee, Y. J.; Kudva, G.; Litzinger, T. A. Combust. Flame 1999, 
(69) Politzer, P.; Boyd, S. Struct. Chem. 2002, 13, 105.

(70) White, C. T.; Robertson, D. H.; Elert, M. L.; Brenner, D. W. In

Microscopic Simulations of Complex Hydrodynamic Phenomena; Mareschal, M., Holian, B. L., Eds.; Plenum Press: New York, 1992; pp 111.

(71) Brenner, D. W.; Robertson, D. H.; Elert, M. L.; White, C. T. Phys. Rev. Lett. 1993, 70, 2174.

(72) Elert, M. L.; Zybin, S. V.; White, C. T. J. Chem. Phys. 2003, 118, 9795.

(73) Bickham, S. R.; Kress, J. D.; Collins, L. A. Journal of Chemical Physics 2000, 112, 9695 .

(74) Kress, J. D.; Bickham, S. R.; Collins, L. A.; Holian, B. L.; Goedecker, S. Physical Review Letters 1999, 83, 3896.

(75) Lewis, J.; Sewell, T.; Evans, R.; Voth, G. J. Phys. Chem. B 2000, 104, 1009.

(76) Smith, G.; Bharadwaj, R.; Bedrov, D.; Ayyagari, C. J. Phys. Chem. B 1999, 103,705 .

(77) Sorescu, D. C.; Rice, B. M.; Thompson, D. L. J. Phys. Chem. B 1998, 102, 6692.

(78) Sorescu, D.; Rice, B.; Thompson, D. J. Phys. Chem. B 1999, 103, 6783.

(79) Elstner, M.; Porezag, D.; Jungnickel, G.; Elsner, J.; Hauk, M.;

Frauenheim, T.; Suhai, S.; Seifert, G. Physical Review B 1998, 58, 7260.

(80) Reed, E.; Joannopoulos, J. D.; Fried, L. Physical Rev. B 2000, 62, 16500.

(81) Wu, C. J.; Fried, L. E. J. Phys. Chem. A 2000, 104, 6447.

(82) Manna, M. R.; Fried, L. J. Phys. Chem. A 1998, 102, 9884.

(83) Wu, C. J.; Yang, L. H.; Fried, L. E.; Quennevill, J.; Martinez, T. J. Phys. Rev. B 2003, 67, 235101.

(84) Rice, B.; Adams, G.; Page, M.; Thompson, D. J. Phys. Chem. 1995, 99, 5016.

(85) Williams, F. Advances in Chemical Physics 1971, 21, 289.

(86) Kuklja, M. M.; Kunz, A. B. J. Appl. Phys. 2000, 87, 2215.

(87) Reed, E. J.; Joannopoulos, J. D.; Fried, L. E. Phys. Rev. Lett. 2003, 90, 235503.

(88) Maillet, J. B.; Mareschal, M.; Soulard, L.; Ravelo, R.; Lomdahl, P. S.; Germann, T. C.; Holian, B. L. Phys. Rev. E 2001, 63, 016121. 